\title{
\begin{tabular}{l|l} 
Mibraries & DSpace@MIT
\end{tabular}
}

\author{
MIT Open Access Articles
}

\section{Photoporomechanics: An Experimental Method to Visualize the Effective Stress Field in Fluid-Filled Granular Media}

The MIT Faculty has made this article openly available. Please share how this access benefits you. Your story matters.

Citation: Li et al., “Photoporomechanics: An Experimental Method to Visualize the Effective Stress Field in Fluid-Filled Granular Media." Phys. Rev. Applied 16 (2020)

As Published: 10.1103/physrevapplied.16.024043

Publisher: American Physical Society (APS)

Persistent URL: https://hdl.handle.net/1721.1/132997

Version: Final published version: final published article, as it appeared in a journal, conference proceedings, or other formally published context

Terms of Use: Article is made available in accordance with the publisher's policy and may be subject to US copyright law. Please refer to the publisher's site for terms of use. 


\title{
Photoporomechanics: An Experimental Method to Visualize the Effective Stress Field in Fluid-Filled Granular Media
}

\author{
Wei Li®, Yue Meng®, Bauyrzhan K. Primkulov®, and Ruben Juanes®* \\ Massachusetts Institute of Technology, 77 Massachusetts Avenue, Cambridge, MA, USA
}

(Received 4 May 2021; revised 19 July 2021; accepted 30 July 2021; published 25 August 2021)

\begin{abstract}
Effective stress governs the mechanical behavior of porous media. In this study, we use photoelasticimetry to visualize the evolving effective stress field in fluid-filled granular media in processes that couple fluid flow and mechanical deformation. We refer to this experimental method as photoporomechanics. We develop a fabrication process to produce millimeter-scale residual-stress-free photoelastic spheres with high geometric accuracy. We use color to quantify the forces acting on the particles over a wide range of forces, while using light intensity for a small range of forces. We then provide an application of photoporomechanics to illustrate the evolution of effective stress during one-dimensional consolidation: a process by which the stresses caused by a sudden load are gradually transmitted through a fluid-filled granular pack as the fluid drains and excess pore pressures dissipate. Our technique provides a powerful experimental model system to study the grain-scale underpinning of coupled solid-fluid processes in granular media.
\end{abstract}

DOI: 10.1103/PhysRevApplied.16.024043

\section{INTRODUCTION}

When stress is applied to porous media, part of the stress is transmitted through the pore fluid and part of the stress is transmitted through the solid skeleton. Effective stress - the fraction of the total stress that is transmitted through the solid skeleton-controls the mechanical behavior of porous media, from land subsidence due to groundwater pumping to the cohesion of sand in sandcastles. Karl von Terzaghi, father of soil mechanics, introduced this concept a century ago [1,2]. In Terzaghi's conceptualization, effective stress is the calculated stress that is "effective" in moving soil or causing displacement. Unveiling effective stress recognizes the powerful coupling among viscous, capillary, and frictional forces within fluid-filled porous media, especially granular media with strongly coupled fluids [3-7]. Porous media physics has experienced profound advances since Terzaghi's work. However, effective stress remains a physical quantity that can only be calculated by subtracting pore pressure from the normal component of the stress tensor, or inferred from its "effect," typically the solid skeleton deformation. Particularly challenging is capturing the evolution of effective stresses in path-dependent physical processes in porous media, such as friction, fracturing, creeping, plastic deformation, and multiphase flows. In this study, we use photoelasticimetry to visualize the evolving effective stress

\footnotetext{
*juanes@mit.edu
}

field in fluid-filled granular media in processes that couple fluid flow and mechanical deformation. We hereby refer to this experimental method as photoporomechanics.

Photoelasticimetry has been used as an experimental method to quantify the internal stresses within solid bodies for several decades [8-13]. This method uses transparent materials that are photoelastic: their degree of birefringence depends on the local anisotropic stresses in the material. When viewed under a polariscope, the stressed photoelastic material shows visual patterns of fringes and colors, which is referred to as photoelastic response. The photoelastic response is correlated with the applied forces or the internal stresses through calibration tests, and then used to visualize and even quantitatively measure the stress field in various mechanical problems [14-35].

This technique, however, has not yet been used to study poromechanical problems because of two major challenges: fabricating three-dimensional (3D) photoelastic particles and obtaining quantitative information on the forces acting on these 3D particles [13,36-38]. Photoelasticimetry is typically implemented using cylindrical disks, which disallow connectivity between the pore space, and therefore cannot be used as an analog of permeable porous media. For a proper analogy of a porous medium regarding the pore geometry, connectivity and morphology [7], a pack of 3D particles, such as spheres, should be used. The particles also have to be small enough that the viscous forces and interfacial forces are sufficiently large to be relevant in the evolution of the solid-fluid system. To date, there has not been a method able to consistently 
fabricate millimeter-scale residual-stress-free 3D particles with acceptable size variations. In addition, 3D particles are lenses by themselves [13], so the distances between the light source, the particle, and the camera determine the observed image. The photoelastic fringes observed on the $2 \mathrm{D}$ disks $[12,13]$ are not observed on $3 \mathrm{D}$ particles, so different methods will be needed to obtain quantitative information on the forces based on the photoelastic response of the 3D particles.

Here we address these two challenges, and establish the methodology of photoporomechanics. We design the fabrication processes, similar to "squeeze casting," and produce millimeter-scale residual-stress-free spheres with high geometric accuracy. We then study the photoelastic response of these spheres under different applied forces. We use color to quantify the forces acting on the particles over a wide range of forces, while using light intensity for a small range of forces. We then provide an application of photoporomechanics to illustrate the evolution of effective stress during 1D consolidation: a process by which the stresses caused by a sudden load are gradually transmitted through a fluid-filled granular pack as the fluid drains and excess pore pressures dissipate. Our technique provides a powerful experimental model system to study the strong coupling of solid and fluid in granular media.

\section{FABRICATION OF PHOTOELASTIC SPHERES}

Our fabrication process differs from existing methods $[12,13,39-41]$, and is instead similar to "squeeze casting" for metals [42], but for polymer resins in our case. It is suitable for almost any castable photoelastic polymer.

The molds used for casting are shown in Fig. 1. The two halves of the mold are identical, each containing arrays of hemispherical pockets. These molds are made of polyoxymethylene, also known as acetal plastic, for its high stiffness, good machinability, and low friction. The mold surface is first machined flat, then milled with a ball end mill to create the hemisphere cavities. With the help of a CNC mill, the geometric tolerance of the mold is controlled within $\pm 1 / 1000^{\prime \prime}( \pm 25 \mu \mathrm{m})$. Alignment pins are used to ensure the two parts mate and create spherical cavities for the resin to fill in.

First, the two halves of the mold are sprayed with mold release agent and assembled with dowel pins, keeping them aligned and leaving a gap for the resin. The mold is then placed in a semirigid plastic bag that closely fits the assembled mold. Resin is mixed and poured into the plastic bag, submerging the mold. The bag containing resin and mold is then deaired in the vacuum chamber for $10 \mathrm{~min}$ to eliminate trapped air bubbles in the resin and mold. After the vacuum, the two parts of the mold are pressed together (squeeze) and transferred from the bag to a leveled surface. Heavy weights $(>20 \mathrm{~kg})$ are applied to the mold

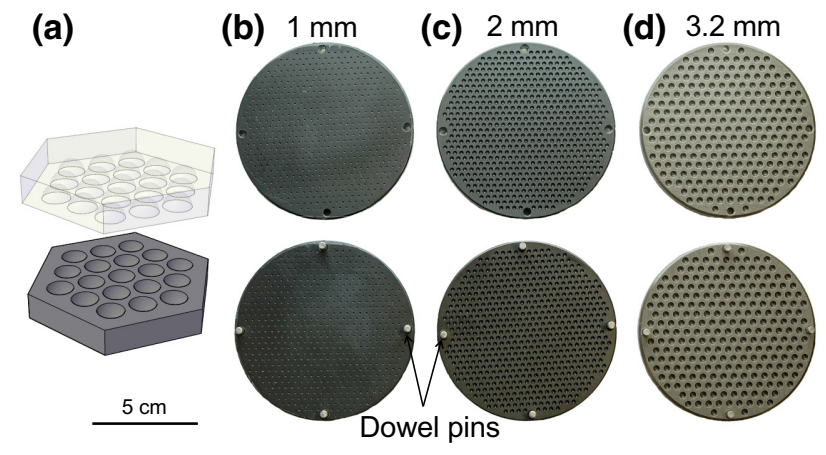

FIG. 1. Molds for squeeze casting. (a) 3D design of the mold. The two halves mate and create spherical cavities for the resin to fill in. (b)-(d) Molds for 1, 2, and $3.2 \mathrm{~mm}$ spheres made of black acetal plastic. Four dowel pins are used for alignment of the two halves. The molds for the three sizes of spheres have a diameter of $3.25 \mathrm{in}$. $(8.26 \mathrm{~cm})$. This size is optimized to be big enough to produce a large number of spheres per mold, and small enough to effectively squeeze out the excess resin in the gap.

to squeeze out the excess resin and reduce the thickness of the thin film around the sphere equator to a minimum. The resin cures in the mold with the weights for the length of time specified by the manufacturer. Then, the alignment pins are removed, and the molds are open to release the photoelastic spheres. Some photoelastic spheres may still have a thin film around their equators, but it tears off easily.

Photoelastic spheres with diameters of 1.0, 2.0, and $3.2 \mathrm{~mm}$ are manufactured using the molds shown in Figs. 1(b)-1(d), respectively. The two-part resin VytaFlex $^{\mathrm{TM}} 20$ from Smooth-On, Inc is used for casting the beads. It produces soft polyurethane spheres with an amber color. A photo of the spheres taken with a polystyrene ruler is shown in Fig. 2(a). The same image taken under the dark-field circular polariscope is shown in Fig. 2(b). The color strips on the polystyrene ruler show that it has residual stress. In contrast, the photoelastic spheres are free of residual stress, and hardly visible in Fig. 2(b). Some reflected light from the spheres' curved surfaces indicates where they are.

To analyze the size and shape of the spheres resulting from the fabrication process, we take images of hundreds of the spheres on a light panel with a ruler as a reference, similar to Fig. 2(a). The images are binarized, filtered, and analyzed to study the particle size distribution and circularity [43]. Although this image analysis is based on $2 \mathrm{D}$ images of the 3D spheres, the random orientations of spheres in the 2D images ensure the measured diameter and circularity are representative of their actual values in $3 \mathrm{D}$. For a binarized image of the sphere, assuming there are $N_{\text {AP }}$ pixels representing it in the image and $N_{\text {PP }}$ pixels representing its perimeter, the equivalent diameter $\left(D_{\mathrm{eq}}\right)$ and 


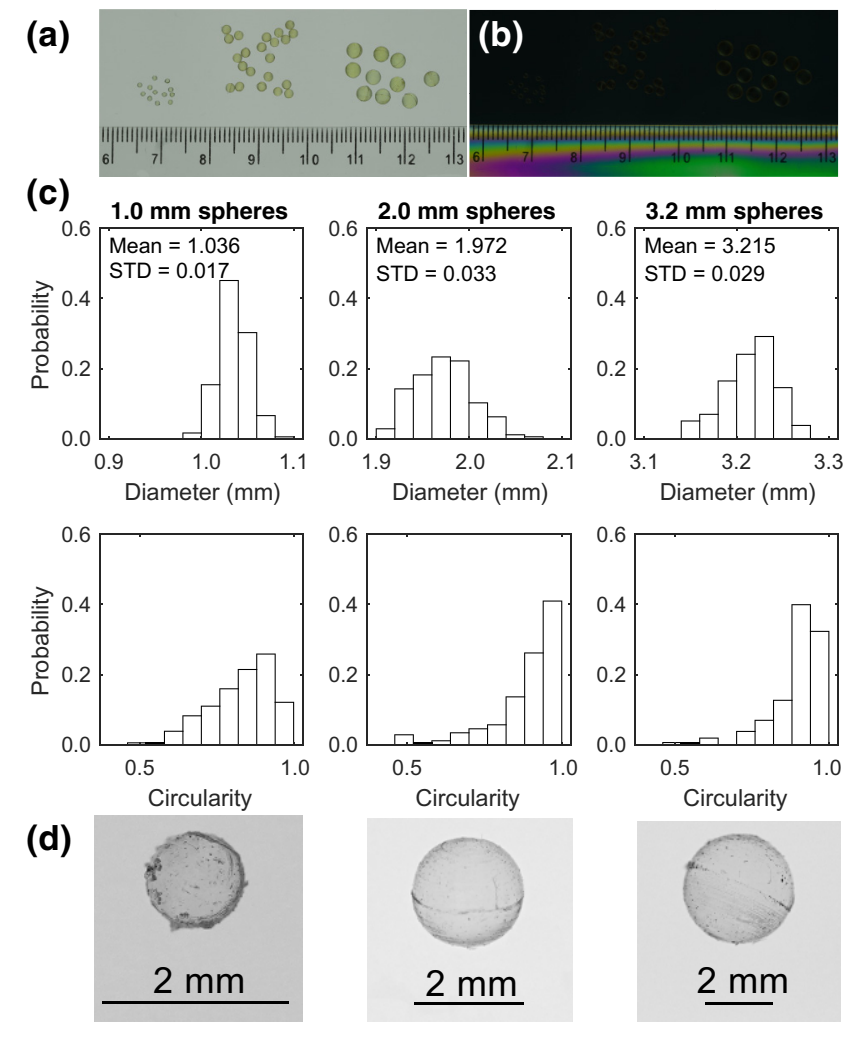

FIG. 2. The resulting photoelastic spheres from the fabrication process. (a) The three sizes of spheres under white light. (b) The three sizes of spheres under a circular polariscope. The polystyrene ruler, having residual stress, shows color strips. However, the spheres, being residual-stress-free, are hardly visible. (c) Size and circularity of the spheres. (d) Macro images of the spheres showing their surface roughness.

circularity $\left(C_{c}\right)$ are defined as

$$
\begin{aligned}
D_{\mathrm{eq}} & =2 S_{c} \sqrt{N_{\mathrm{AP}} / \pi}, \\
C_{c} & =\frac{4 \pi N_{\mathrm{AP}}}{N_{\mathrm{PP}}^{2}},
\end{aligned}
$$

where $S_{c}$ is the scale of the image with a unit of length per pixel. The circularity is one of the often-used shape factors to quantity how close to a circle a shape is [43]. A circle, having the largest area for a given perimeter, has a circularity of 1 . Any other shape has circularity less than 1. The diameter and circularity calculated in this method are summarized in Fig. 2(c) for the three sizes of spheres. The diameters of the spheres have a narrow distribution, with a standard deviation less than $5 \%$ of the mean. The circularities of the three sizes of spheres are close to 1 .

Macro images of the spheres are shown in Fig. 2(d). The photoelastic spheres all have lines around their equators. These result from the residual film between the two molds of the squeeze casting. The surface roughness of the spheres results from the machine marks on the mold, which is at the scale of microns. On smaller spheres, this surface roughness is relatively more pronounced than that on larger spheres.

\section{PHOTOELASTIC RESPONSE OF THE SPHERES}

Under stress, the photoelastic spheres do not exhibit photoelastic fringes [37,44], because the light refracts through the angled surface near the contact points. The well-established fringe inversion algorithm for vector measurements of forces based on fringes on 2D disks [10] cannot be used for spheres. Neither is it possible for thousands of millimeter-scale spheres. As used by other studies for 2D disks [12,13], we use light intensity for scalar measurements of forces on spheres, which works for a narrow range of forces. To extend the range of forces measurable using the photoelastic spheres, we use color to quantify the photoelastic response, and determine the applied forces.

When a photoelastic sphere is subject to stress, it becomes birefringent and splits the light through the sphere into two beams with different refractive indices $n_{1}$ and $n_{2}$. After exiting the sphere, the two beams have a retardation $(\gamma)$, or the so-called path difference:

$$
\gamma=\tau\left(n_{2}-n_{1}\right)
$$

Here $\tau$ is the thickness of the material. These two beams of light interfere at the left circular polarizer (Fig. 3) and show intensity or color changes as a function of the path difference. For monochromatic light, the result of light interference is the periodic increase and decrease of light intensity with increasing path difference. For white light, the result of interference is the color change as a function of the path difference, as shown with the Michel-Lévy birefringence chart [45]. With the increasing force, the photoelastic sphere's birefrengence increases and leads to a larger path difference. The resulting color can be found along the Michel-Lévy chart, which also indicates that the light intensity first increases and then decreases. While the colors shown on the photoelastic spheres should be similar to those on the Michel-Lévy chart, in practice they can be different due to the light source, filters, and the color of the material itself. We designed a calibration setup to correlate the force on the sphere and its photoelastic response, as shown in Fig. 3. For this study, the $2 \mathrm{~mm}$ spheres are used.

This calibration setup is configured as a load-framecoupled dark-field circular polariscope [Fig. 3(a)]. We use a white light LED panel as the light source. Two sets of linear polarizers and quarter-wave plates configure the dark-field circular polariscope. A high-resolution (60 MP) color camera is used to take the images of the photoelastic spheres. The computer-controlled load frame moves the platen to the accuracy of $\pm 0.6 \mu \mathrm{m}$, and measures the vertical displacements and forces. Figure 3(b) shows a close-up 


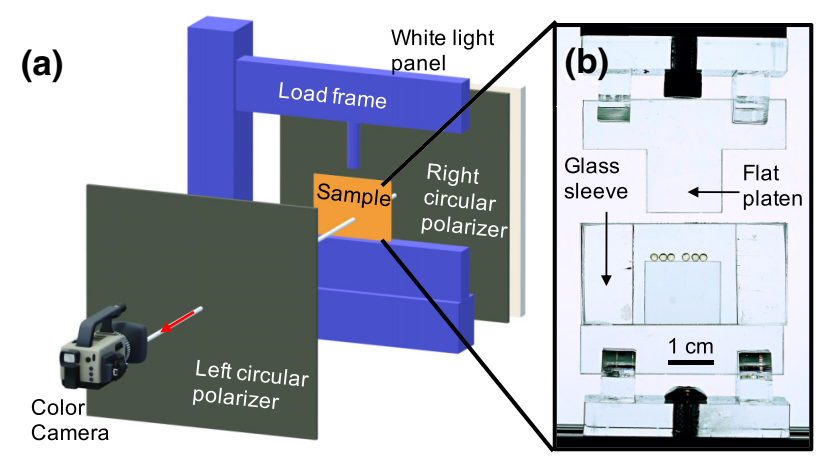

(c)

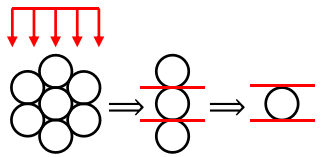

(d) $\square \downarrow \downarrow$
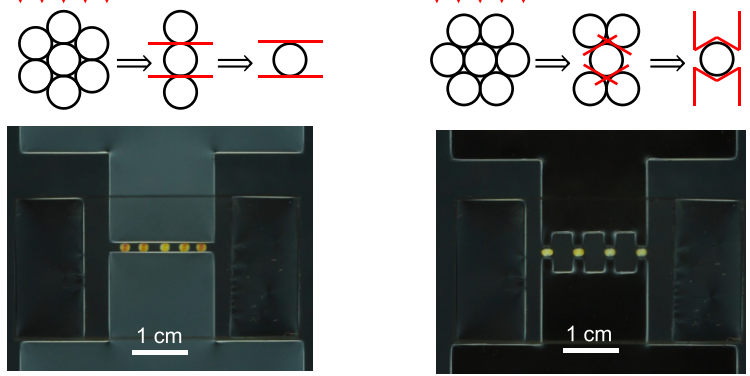

FIG. 3. Experimental setup to study the photoelastic response of $2 \mathrm{~mm}$ spheres. (a) A load-frame-coupled dark-field polariscope. (b) A close-up of the $2 \mathrm{~mm}$ spheres between the flat platens. (c) Spheres between two flat platens. (d) Spheres between two $120^{\circ}$ platens.

of the platens with the photoelastic spheres. A glass sleeve with a $2.1 \mathrm{~mm}$ aperture is used to keep the spheres on the bottom platen. The top and bottom platens are made of laser-cut $1.8 \mathrm{~mm}$ acrylic sheets. We use two types of platens to simulate the loading conditions of single spheres in a monolayer of spheres under vertical load, as shown in Figs. 3(c) and 3(d). The sphere packs both have a hexagonal packing, but in different orientations relative to the vertical load. We refer to the hexagonal packing in Fig. 3(c) as vertical hexagonal packing (VHP), because there are vertically aligned spheres. Similarly, we refer to the hexagonal packing in Fig. 3(d) as horizontal hexagonal packing (HHP), which has a $30^{\circ}$ rotation from VHP because of the rotational symmetry of the two packings. Since the vertical load in VHP is transmitted mostly vertically along the spheres, the sphere at the center is loaded by the top and bottom spheres with horizontal contact lines [red in Fig. 3(c)]. Hence, the loading condition of a single sphere in VHP can be approximated by two flat platens. In contrast, the vertical load is transmitted to the sphere at the HHP center through the upper and lower sphere pairs each with $30^{\circ}$ contact lines relative to the horizon. Therefore, the loading condition for the sphere in the HHP center can be approximated by two $120^{\circ}$ platens.
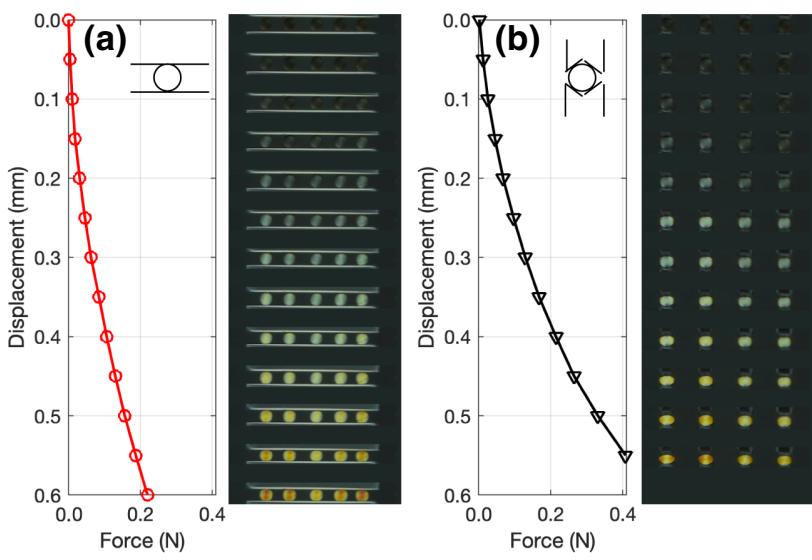

(c)

Michel-Lévy Chart

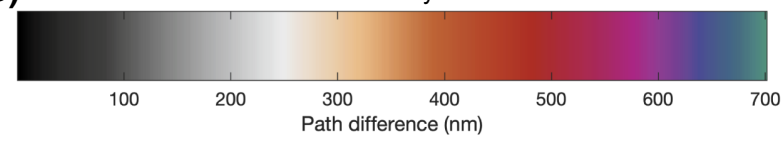

FIG. 4. Photoelastic response of the spheres. The average force on each sphere is plotted against the displacement of the platen. The photos of the spheres are aligned with the forcedisplacement steps. (a) Loading the spheres with flat platens. (b) Loading the spheres with $120^{\circ}$ platens. (c) Reconstructed first $700 \mathrm{~nm}$ of the Michel-Lévy chart. This color evolution of the spheres under increasing load is similar to the interference colors along the Michel-Lévy chart. See the videos for the two calibration tests within the Supplemental Material [46].

The calibration tests are conducted by loading and unloading several spheres in steps, while recording their photoelastic responses. For each load step, the top platen moves downward $0.05 \mathrm{~mm}$ and holds its position, while the force and photo of the spheres are recorded. Figures 4(a) and 4(b) show the results of the calibration tests, in which the photos of the spheres are aligned with the forcedisplacement steps. The forces in Figs. 4(a) and 4(b) are the measured force averaged over the number of spheres used in the test. Only the loading part is shown for simplicity in Fig. 4. The loading-unloading cycle is shown in the videos for the two tests within the Supplemental Material [46]. During loading, the reaction forces on the spheres increase, while the colors of the spheres change from black to bright and orange. This color evolution of the spheres under increasing load is similar to the interference colors along the Michel-Lévy chart shown in Fig. 4(c). However, because of the light source, filters, and the color of the material, the colors differ from the Michel-Lévy chart by having a green tint.

In this study, we use two methods to correlate the sphere's photoelastic response with the applied load. The first method is to correlate the light intensity with the force acting on the sphere. This is the simplest way to use photoelastic material for force measurement [47]. Figure 5(a) shows the light intensity of the spheres under the two 

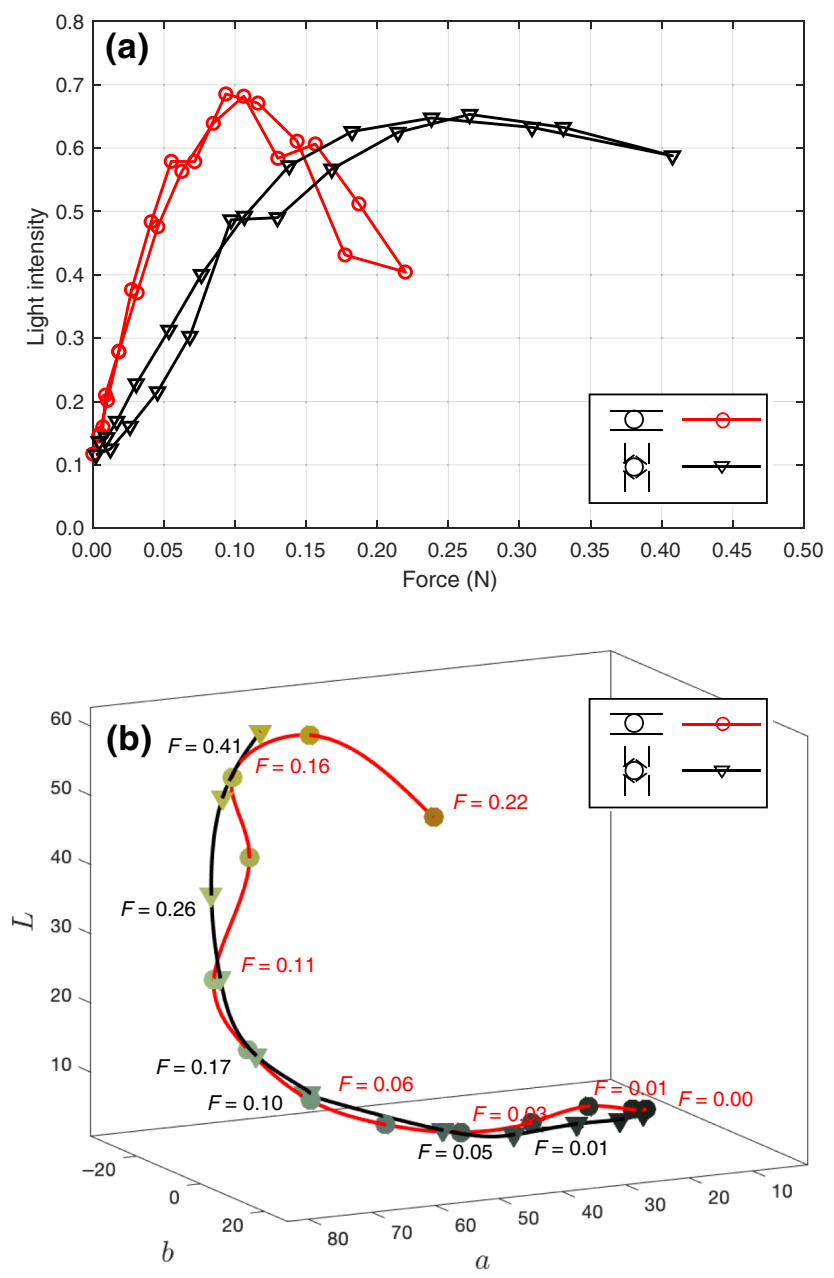

FIG. 5. Correlating the photoelastic response with the load. (a) The light intensity of the spheres are plotted with the forces for the two types of loading conditions. (b) The colors of the spheres are plotted in the CIELAB color space with the forces near them.

loading conditions. For small forces $(0 \sim 0.1 \mathrm{~N})$, a linear relation can be used to relate forces to the sphere light intensities. However, the same light intensity indicates a larger force in HHP than that in VHP. For larger applied forces, the relation between the light intensity and force becomes nonmonotonic. Therefore, using the light intensity to measure contact forces is only suitable for a small range of forces $(0 \sim 0.1 \mathrm{~N})$.

The second method is to use the color information to cover a larger range of loads, since the evolution of color with increasing force follows a unique path. This path is presented using the CIELAB color space [Fig. 5(b)]. CIELAB was intended as a perceptually uniform space, where a given numerical change in the $L, a$, and $b$ axes corresponds to a similar perceived change in color [48]. As shown in Fig. 5(b), the sphere colors for both loading conditions evolve as the loads increase, following almost the same path in the CIELAB space. However, the same color indicates a larger force in HHP than that in VHP. Nevertheless, using the CIELAB color space, we provide a one-to-one mapping between the color and force. To predict the force based on the color, the $(L, a, b)$ coordinates of this color can be used to find the closest point on the curves shown in Fig. 5(b) for its corresponding force.

The above discussion demonstrates two methods to correlate the spheres' photoelastic response with the applied load using the $2 \mathrm{~mm}$ VytaFlex ${ }^{\mathrm{TM}} 20$ polyurethane spheres. For these photoelastic spheres, light intensity can be used to quantify a small range of forces $(0 \sim 0.1 \mathrm{~N}$ for VHP $)$, while color can be used to quantify a large range of forces $(0 \sim 0.22 \mathrm{~N}$ for VHP $)$. However, the range of forces measurable using these two methods is case specific, depending on the stiffness, size, and birefrengence property of the material. The bit depth, noise ratio, and sensitivity of the camera sensor determine the precision and sensitivity of these measurements. For example, if a 12-bit monochrome sensor with a 2-bit typical noise is used to map out the full range of light intensity change in the calibration test on VHP, the theoretical precision of this measurement is $0.1(\mathrm{~N}) /\left(2^{(12-2)}-1\right) \sim 0.1 \mathrm{mN}$. Color sensors are often 24-bit, offering more bit depth. However, the theoretical precision of force measurement using color is difficult to estimate because of the various color filter arrays and interpolation algorithms used in different cameras. Therefore, calibration tests, like those presented in this section, should be done with the light source, filters, photoelastic materials, and cameras used in the photoporomechanics test for a case-specific correlation.

Our study of the photoelastic response is intended, like in the traditional use of photoelasticimetry $[8,12,13]$, for quasi-2D monolayer experiments, where the polarized light is transmitted perpendicularly through the $2 \mathrm{D}$ plane defining the monolayer of spheres. This ensures that the observed light intensity- or color change at each location is caused by one sphere only, and thus can be associated with the force that is exerted on that sphere. In addition, the calibration process is conducted at the level of a single sphere with simulated loading conditions and packing orientations, and thus may not be directly applicable for a pack of spheres. The best practice is to associate the light intensity or color with the known forces in the experimental setup for the test of interest, as done in the consolidation test discussed in Sec. IV.

\section{PHOTOPOROMECHANICS: AN EXAMPLE}

With the two challenges of photoporomechanics addressed in the previous two sections, we use the photoelastic spheres to assemble a monolayer porous medium and study a classic poromechanical problem: 1D consolidation $[1,2]$. While this is the paradigmatic example of coupled flow and deformation in a porous medium, and has served as the basis for the concept of effective 
stress, the effective stress has heretofore remained elusive to direct experimental quantification - something that our photoporomechanics technique allows us to resolve.

\section{A. Consolidation tests}

We designed a monolayer consolidation cell to conduct the consolidation test [Fig. 6(b)]. Two thick glass plates are glued with a $2 \mathrm{~mm}$ thick U-shaped spacer to form a monolayer cell. The inner dimensions of the cell are $100 \mathrm{~mm}$ in the horizontal direction $(L), 100 \mathrm{~mm}$ in the vertical direction $(H)$, and $2.1 \mathrm{~mm}$ as the aperture between the two glass plates $(T)$ because of the thickness of the glue. A hole is drilled at a distance of $2 \mathrm{~mm}$ from the inner bottom of the cell to measure fluid pressure. A fluid reservoir is installed on the top of the cell to provide a constant fluid pressure boundary condition. A piston, made of a $1.8 \mathrm{~mm}$ acrylic plate is used to load the fluid-saturated photoelastic sphere pack. The piston has a dome head to ensure vertical centric load on the piston. Slots are cut into the piston to increase its permeability.

We converted the load frame to provide a constant vertical load, as shown in Fig. 6(a). A stainless steel shaft is used as the weight $(W=5.0 \mathrm{~N})$ to provide a constant load to the consolidation piston. This weight provides a vertical stress of $\sigma_{0}=W /(T L)=23 \mathrm{kPa}$. Two linear bearings are installed in a tubular fixture to limit the shaft's movement to vertical displacement only. We use a linear variable differential transformer (LVDT) to measure the vertical displacement of the piston, and a pressure sensor to measure the pressure at the bottom of the cell.

Each test starts with packing the photoelastic spheres in the cell to a $20 \mathrm{~mm}$ height $(H)$, and follows by saturating the pack with silicone oil. The assembled consolidation cell is then deaired in the vacuum chamber for $10 \mathrm{~min}$ to remove the dissolved and trapped air in the cell. After the vacuum, the consolidation cell is installed in the fixture of the load frame. The shaft is first moved very close to the piston through the linear bearings, then released to load the piston, while the displacement, pore pressure, and video of the pack (see [46]) are recorded. Five types of silicon oils with different viscosities $(10000,5000,1000,500$, and $50 \mathrm{cSt}$ ) are used in the consolidation tests.

The measured displacements and pore pressures for the five tests are shown in Figs. 6(b) and 6(c). Upon application of the load, the pack starts to deform, while the deformation rate depends on the viscosity of the pore fluid-lower viscosity results in faster deformation. Because of the experimental variations in the pack height $(H)$ in every test, the final displacements are different. Upon applying the load, the excess pore pressure immediately builds up to a value approximately equal to the applied stress $(23 \mathrm{kPa})$, then dissipates faster for lower viscosity pore fluid. The behavior of the fluid-saturated photoelastic sphere pack shows the strong coupling between the solid and fluid. When the viscosity of the pore fluid is low enough, the excess pore pressure dissipates so fast that not much excess pore pressure can be measured, and the pack deforms almost immediately $(<1 \mathrm{~s})$.
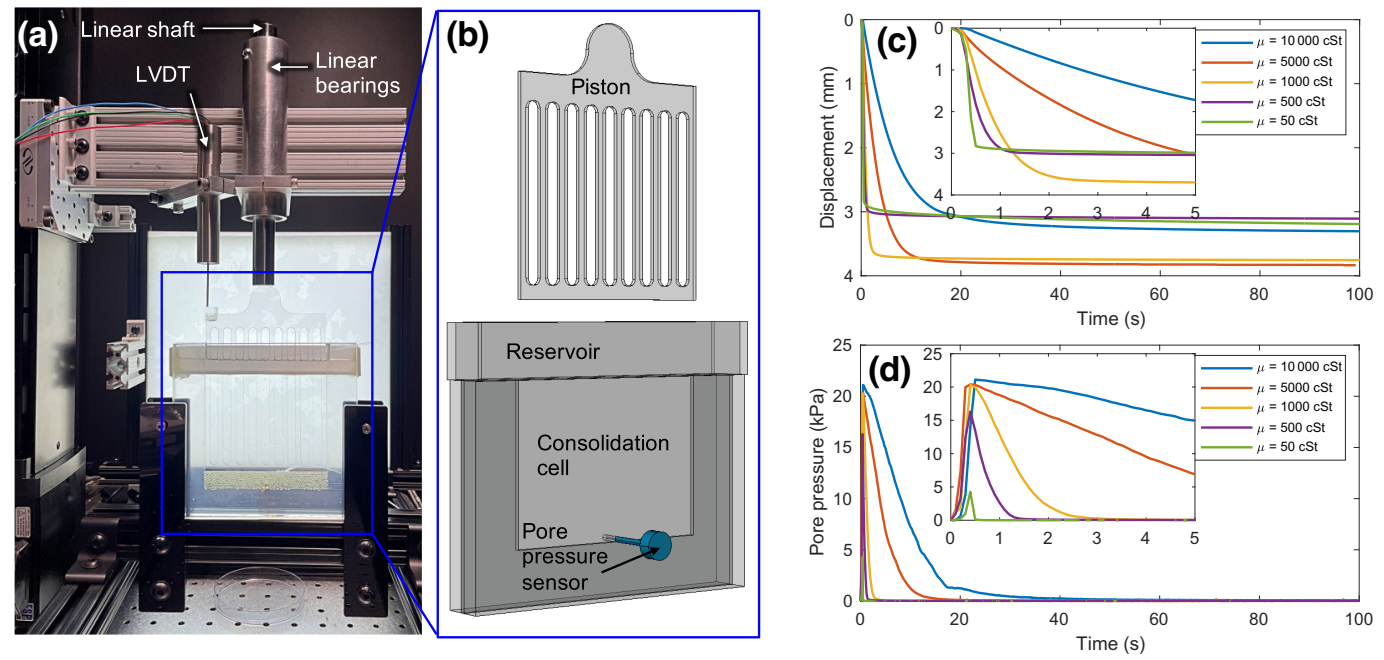

FIG. 6. 1D consolidation test. (a) Experimental setup for the 1D consolidation setup. The pack of fluid saturated photoelastic spheres are loaded suddenly with a constant weight, while the video, deformation, and excess pore pressure are recorded. (b) Detailed schematic of the consolidation cell. Two thick glass plates are glued with a $2 \mathrm{~mm}$ thick U-shaped spacer to form a monolayer cell. The excess pore pressure is measured at the bottom of the cell with a pressure sensor. The pore fluid is filled to the middle of the reservoir to provide a constant-pressure boundary condition. A piston is made of a $1.8 \mathrm{~mm}$ acrylic plate with slots cut out to increase its permeability in the cell. (c) Displacement of the piston. (d) Excess pore pressure measured by the pressure sensor. 


\section{B. 1D consolidation model}

The 1D consolidation tests use a piston with a permeability comparable to that of the pack of spheres, as measured in Appendix A and listed in Table I in Appendix C. The presence of this piston causes an additional pressure drop as the fluid that drains from the sphere pack flows through it. The 1D consolidation model accounting for the comparable piston permeability is derived in Appendix B. The piston, having low permeability but high stiffness, is modeled as a Robin boundary condition, as discussed in Appendix B. This model is different from the classic 1D consolidation model $[2,49]$, which has a constant-pressure (Dirichlet) boundary condition.

The experimental parameters used in the model are summarized in Table I in Appendix C. The 1D volumetric modulus of the pack $(M=300 \mathrm{kPa})$ is measured using the load frame, similar to the calibration tests done in Sec. III. The permeability of the monolayer sphere pack $k$ can be estimated using the KozenyCarman equation as $\left(0.03 d_{s}\right)^{2}$ [50]. In the experiment, we choose the value $3.0 \times 10^{-9} \mathrm{~m}^{2}$ to fit the experimental results. The experimental parameters result in the permeability ratio $C_{d}=\left(k_{p} H\right) /\left(k L_{p}\right)$ of 1.02 , which shows that the permeabilities of the pack and piston are indeed comparable.

The results of the three tests with the high fluid viscosity are compared with the analytical solution in Fig. 7. The time and measured pore pressure are converted to dimensionless form [Eq. (B7)]. The measured piston displacement is converted to the degree of consolidation [Eq. (B14)]. By converting the experimental measurements to dimensionless quantities, the test results show one consistent trend, which matches the prediction of the 1D consolidation model with Robin boundary condition. Comparing with the classic 1D consolidation theory (Dirichlet boundary), the $1 \mathrm{D}$ consolidation with Robin boundary condition has a slower pore pressure dissipation, as shown in Fig. 7.
The comparison between the experimental results and the 1D consolidation model shows that the pack of photoelastic spheres exhibits strong coupling with the pore fluid, and can be used to study poromechanical problems. The predictions from the model of 1D consolidation with Robin boundary condition agree well with the experimental results. However, the model slightly underestimates the excess pore pressure (Fig. 7). This small discrepancy is due to the model's assumption of constant pack height and piston length. As the pack is being compressed, its permeability decreases, while the length of the piston increases. Both of these factors contribute to slowing down the excess pore pressure dissipation, but they are not considered by the model.

\section{Visualizing the evolving effective stresses}

The photoelastic spheres in this pack can be used to directly observe the evolution of the forces in the solid phase. We use the test with $\eta=5000 \mathrm{cSt}$ to study these forces during consolidation. The degree of consolidation [Eq. (B14)] is plotted with the six corresponding frames from the recorded video in Figs. 8(a) and 8(b). After the load is applied, it takes the pack about $10 \mathrm{~s}$ to reach $95 \%$ of the consolidation. The load is transferred down to the pack in the form of force chains, which are chain- or networklike structures composed of stressed spheres.

To enhance the visual effect of these force chains, we first convert the images in Fig. 8(b) to grayscale images, then zero the part that has lower light intensity than the initial frame (not shown in Fig. 8). The resulting grayscale image is rendered with a "hot" color profile for high visual contrast, as shown in Fig. 8(c). As the pore pressure dissipates from the top boundary, the stress chains start to develop from the top boundary, then progresses downwards through the pack. Despite our best effort to pack the sphere uniformly in the cell, the force chains show that the packing in the center of the cell is denser; thus, the earlier emergence of force chains. Because of the narrow range of
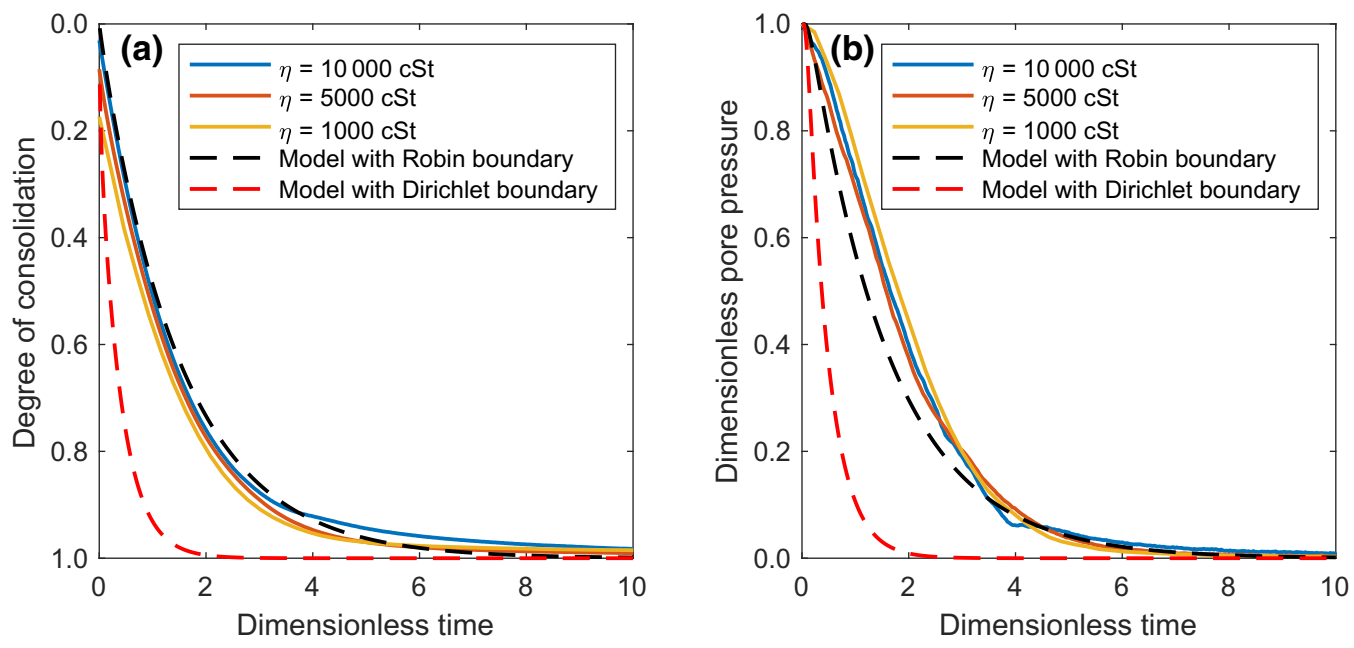

FIG. 7. Experimental results compared with predictions of the 1D consolidation models. (a) Degree of consolidation calculated based on the piston displacement [Eq. (B14)]. (b) Dimensionless excess pore pressure. The experimental results of the three tests show one consistent trend, which matches the prediction of the 1D consolidation model with Robin boundary condition. 

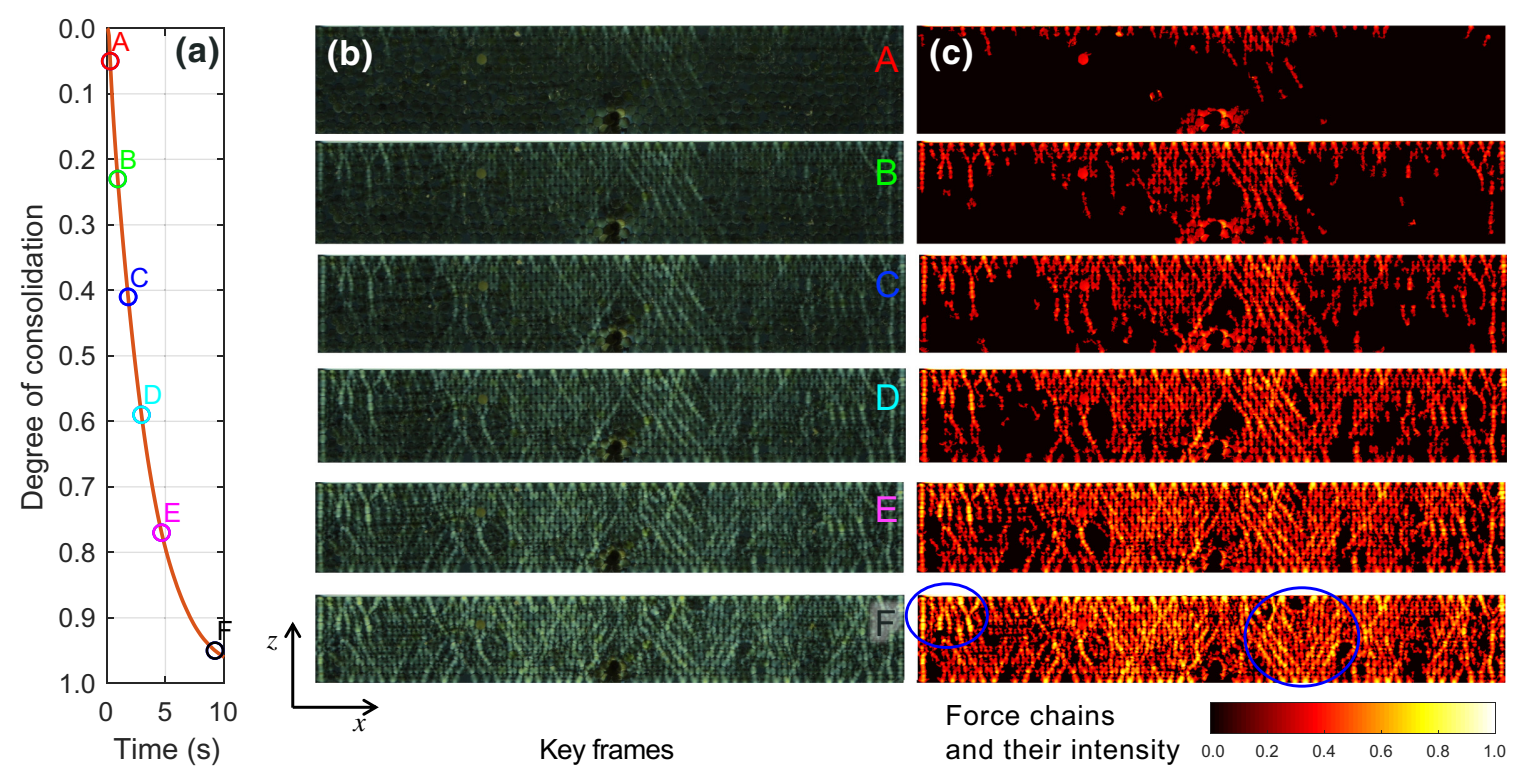

FIG. 8. Photoelastic response of the pack as the excess pore pressure dissipates. (a) The normalized displacement curve for the consolidation test with $\eta=5000 \mathrm{cSt}$. (b) The six snapshots during the consolidation tests. (c) Enhanced images to study the evolution of force chains. The force chains develop from the top boundary, then progresses downwards through the pack. The pack crystallizes into several patches with different orientations, as circled in blue. See the video for the consolidation test in real time within the Supplemental Material [46].

sphere diameters, the pack crystalized into several patches, as the blue circles indicated examples in Fig. 8(c). Based on the geometry of the force chains, the orientation of the hexagonal packing can be identified. The circled patch on the left is VHP, while the circled patch on the right is HHP.

In a continuum framework of poromechanics, the sphere pack is the load-bearing skeleton. The photoelastic response of the spheres then reflects the stress in the solid skeleton, which is the effective stress. The consolidation test, with its known initial and final stress conditions, can be used to correlate the photoelastic response with the effective stress. The images in Fig. 8(b) are converted to grayscale with light intensity field, $I(x, z, t)$. Since the consolidation is a $1 \mathrm{D}$ problem, we can use the mean of the light intensity in the $x$ direction, $I(z, t)$. This averaging also homogenizes the patches of spheres that have different orientations of packing. The initial light intensity $[I(z, 0)]$ corresponds to the initial vertical effective stress $\left[\sigma_{z z}^{\prime}(z, 0)=0\right]$, while the final light intensity $[I(z, \infty)]$ corresponds to the final vertical effective stress $\left[\sigma_{z z}^{\prime}(z, \infty)=\right.$ $\left.\sigma_{0}\right]$. In the consolidation tests, the applied load ( $W=$ $5.0 \mathrm{~N}$ ) averaged over the number of spheres in the horizontal direction $(100 \mathrm{~mm} / 2 \mathrm{~mm}=50)$ did not exceed the linear monotonic range $(0 \sim 0.1 \mathrm{~N})$ [Fig. $5(\mathrm{a})$ ]. Therefore, a linear relation can be used to correlate the light intensity and effective stress: $I(z, t) \propto \sigma_{z z}^{\prime}(z, t)$. The light intensity $I(z, t)$ can be nondimensionalized as

$$
I_{D}(z, t)=\frac{I(z, t)-I(z, 0)}{I(z, \infty)-I(z, 0)} .
$$

If the vertical effective stress is also normalized by the applied stress, $\sigma_{D}=\sigma^{\prime} / \sigma_{0}$, the two normalized quantities then have the relation

$$
I_{D}(z, t)=\sigma_{D}(z, t) .
$$

Equation (4) shows that the normalized light intensity of the sphere pack is equal to the normalized vertical effective stress in the pack. Through this correlation, photoelasticimetry can now be used to measure effective stress in a porous medium.

Figure 9 shows the normalized vertical effective stress measured using photoporomechanics and predicted by the 1D consolidation model with Robin boundary condition. The solid lines are the experimental measurements using photoporomechanics $\left(I_{D}\right)$ for the corresponding six frames in Fig. 8(b), as indicated using real time $t$. The normalized effective stresses $\left(\sigma_{D}\right)$ predicted using the 1D consolidation model with Robin boundary condition are also plotted in Fig. 9, as indicated using dimensionless time $T_{D}$. Both results capture the trend that the effective stress is higher near the upper boundary $(Z=1)$ and lower near the bottom boundary $(Z=0)$. The two also show that the effective stresses increase as the pore pressure dissipates with time. Because of the additional pressure drop caused by the piston, the excess pore pressure on the upper boundary is not zero. It decreases as the excess pore pressure dissipates. This is reflected by the increase of effective stresses, as indicated by the experimental and modeling results. 

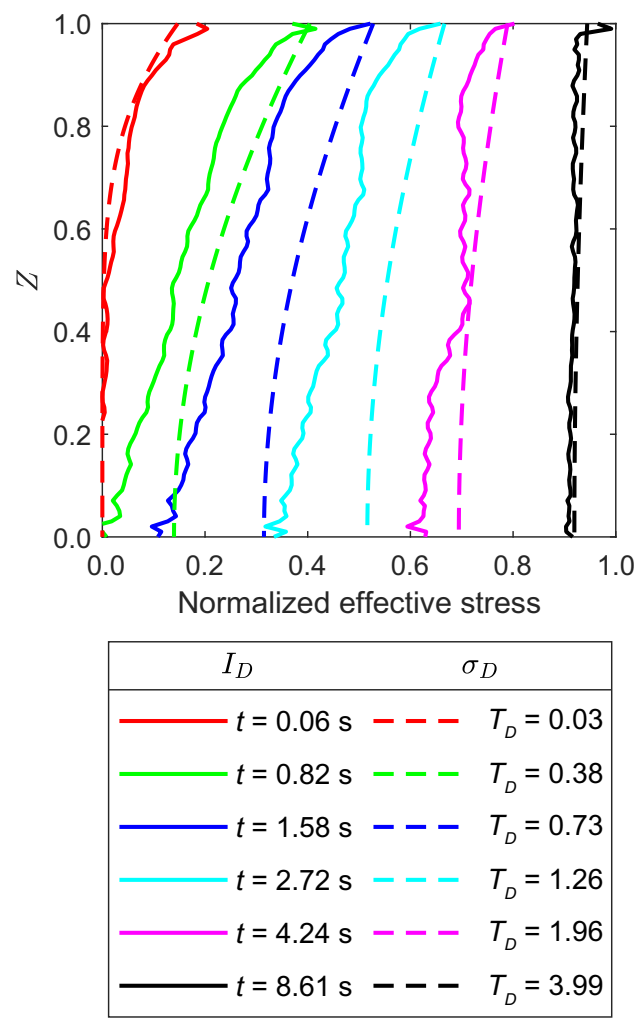

FIG. 9. Evolution of the vertical effective stress. Solid curves represent the measurement based on photoporomechanics. The dashed curves represent the predictions made by the $1 \mathrm{D}$ consolidation model with Robin boundary condition.

There are some discrepancies between the two results, as indicated by the curves B, C, and D in Fig. 9(c). The model predicts higher effective stresses than the experimentally measured effective stress. This is due to the model's underestimated pore pressures shown in Fig. 7(b). As the pack is being compressed, its permeability decreases, while the length of the piston increases. These two factors both slow the excess pore pressure dissipation; effects that are not accounted for in the model. This underestimated pore pressure results in overestimated effective stresses compared to the experimental results.

\section{CONCLUSIONS}

In summary, we have developed an experimental methodology, which we call photoporomechanics. In so doing, we overcome the two challenges of applying photoelasticimetry to fluid-coupled granular media: fabricating 3D photoelastic particles and obtaining quantitative information on the forces applied on these particles. On the one hand, our "squeeze casting" process produces millimeter-scale residual-stress-free photoelastic spheres with high geometric accuracy. On the other hand, we employ a calibration technique to determine interparticle forces, which uses stress-dependent particle color; this calibration technique is applicable over a wider range of forces than the more classical calibration based on light intensity.

These advances enable experimental investigations of the coupling between fluid flow and solid mechanics in granular media. In particular, we can now directly observe the evolution of effective stress - the fraction of applied stress in a porous medium that is transmitted through the solid skeleton via interparticle forces. While effective stress is a fundamental quantity that controls deformation and failure of porous media, it had heretofore remained inaccessible to direct measurement in a granular pack. We demonstrate the ability to quantify the evolution of effective stress with a variant of the classical 1D consolidation, in which a load is applied suddenly to a fluid-filled granular pack, causing delayed deformation as the fluid seeps out of the porous medium. We show that compaction of the granular pack is concomitant with the emergence of particle-particle force networks, which originate at the top boundary (where the pore fluid seeps out) and propagate downwards through the pack as the pore pressure gradually dissipates.

Beyond its intrinsic interest for direct visualization of effective stress in granular matter filled with single-phase fluids, our technique provides more generally a methodology to study the grain-scale underpinning of coupled fluid-solid processes in granular media [51,52], including capillary cohesion [53], desiccation cracks [54,55], capillary fracturing $[6,56]$, gas venting [57-59], frictional flows [4], flow-induced aggregate deformation [60-62], and fluid-injection induced fault slip [63-65].

The isotropic geometry of our photoelastic spheres also makes it possible to apply photoporomechanics in 3D granular systems. Imaging techniques, such as index matching, fluorescence, confocal microscopy, and tomography, can be combined with photoporomechanics to visualize the 3D effective stress field in coupled fluid-granular systems. By providing a wealth of microscopic observables in $3 \mathrm{D}$, including contact forces, length and orientation of force chains, particle coordination number, and stick-slip behavior, this promising experimental technique could revolutionize our understanding of granular systems, which has, for decades, been derived from 2D experimental investigations.

\section{ACKNOWLEDGMENTS}

We thank Professor Brian Evans and Dr. Ulrich Mok for helpful discussions and suggestions. We appreciate Stephen Rudolph for his precision making of the molds. This work is funded by the US Department of Energy (Grant No. DE-SC0018357). 


\section{APPENDIX A: PERMEABILITY OF THE PISTON}

The permeability of the piston is determined by measuring its terminal velocity $\left(v_{\mathrm{pt}}\right)$ in the cell filled with the viscous silicon oil. The piston falling in the cell causes the fluid to leave the bottom of the cell with rate

$$
Q_{f}=v_{\mathrm{pt}} A_{p},
$$

where $A_{p}$ is the cross section area of the piston. The pressure applied to the fluid at the bottom of the piston is the weight of the piston plus the LVDT rod that sits on the piston over the area of fluid:

$$
P_{p}=\frac{W_{p}}{L T}
$$

Assuming that the piston has a section of length $L_{p}$ in the cell, the overall pressure gradient of the fluid can be calculated as $P_{p} / L_{p}$. Although the piston has slots to increase permeability, here we assign its overall permeability as $k_{p}$. Then Darcy's law produces the relation

$$
Q_{f}=L T \frac{k_{p}}{\eta} \frac{P_{p}}{L_{p}}
$$

Therefore, the overall permeability of the piston can be calculated as

$$
k_{p}=\eta v_{p t} \frac{L_{p} A_{p}}{W_{p}} .
$$

The length $L_{p}$ increases as the piston falls. Since the length $L_{p}$ is much greater than its travel distance during the tests, $L_{p}$ is assumed to be constant. For the five types of silicone oils used in the test, the four with the highest viscosity produce a steady-state piston terminal velocity measurement. With these velocity-viscosity pairs, the overall permeability of the piston is $1.2 \times 10^{-8} \mathrm{~mm}^{2}$.

\section{APPENDIX B: ONE-DIMENSIONAL CONSOLIDATION WITH ROBIN BOUNDARY CONDITION}

Section B shows that the permeability of the piston needs to be accounted for when modeling the consolidation of the fluid saturated photoelastic spheres. Because of this relatively low permeability, the boundary condition for the photoelastic spheres is no longer a constant pressure boundary, like the classic case. Instead, the piston causes an extra pressure drop that is proportional to the flow rate through the piston. This is a Robin boundary condition and needs to be analytically modeled.

The governing equation for $1 \mathrm{D}$ consolidation is derived based on mass conservation of the incompressible pore fluid:

$$
\frac{\partial \epsilon_{v}}{\partial t}=-\frac{k}{\eta} \frac{\partial^{2} P}{\partial z^{2}}=\frac{1}{m_{v}} \frac{\partial \sigma_{z z}^{\prime}}{\partial t}
$$

Here $\epsilon_{v}$ is the volumetric strain; $k$ is the permeability of the sphere pack; $\eta$ is the viscosity of the fluid; $m_{v}$ is the 1D volumetric modulus of the sphere pack; and $\sigma_{z z}^{\prime}$ is the vertical effective stress. Since the sphere pack is confined in the two horizontal directions, the 1D volumetric modulus equals the 1D confined drained modulus. In the context of discussion, compression is positive. According to Terzaghi [2], the effective stress and pore pressure has the relation $\sigma_{z z}^{\prime}+P=\sigma_{0}$. Therefore, Eq. (B1) can be written for the excess pore pressure $P$. This $1 \mathrm{D}$ consolidation problem is essentially a diffusion problem for the excess pore pressure, i.e.,

$$
\frac{\partial P}{\partial t}=c_{v} \frac{\partial^{2} P}{\partial z^{2}}
$$

where $c_{v}$ is the consolidation coefficient,

$$
c_{v}=\frac{k}{\eta} m_{v}
$$

The consolidation coefficient $\left(c_{v}\right)$ reflects the rate of the excess pore pressure diffusion process. The process is faster with higher permeability pack, lower viscosity fluid, and stiffer pack. For the piston however, the modulus is of the order of a gigapascal; thus, the consolidation process in the piston can be treated as immediate. Figure 10 shows the analogous problem in geomechanics, where two layers of geomaterials with comparable permeability but different moduli are under the total stress $\sigma_{0}$. The layer with low modulus will go through the consolidation process while the layer with high modulus will just experience Darcy flow [49]. The excess pore pressure is shown in Fig. 10(b).

The piston and pack interface satisfies pressure continuity. We denote this excess pore pressure as $P_{1}$. The flux

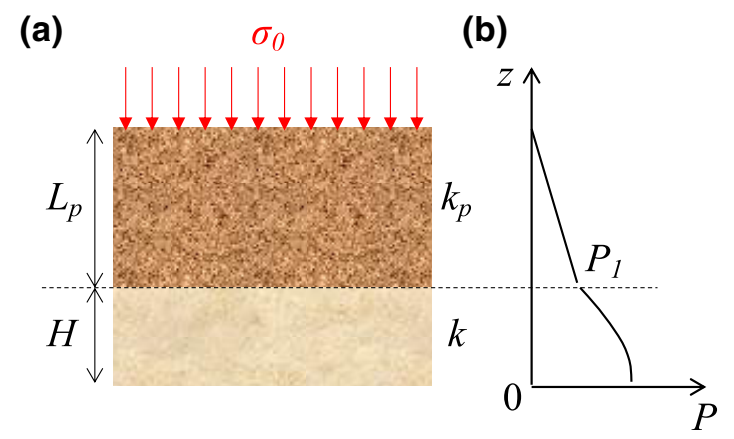

FIG. 10. Analog of the 1D consolidation problem with multiple layers of comparable permeability but different stiffness. 
through the piston $(q)$ can be modeled using Darcy's law:

$$
q=\frac{k_{p}}{\eta} \frac{P_{1}}{L_{P}}
$$

Here $L_{p}$ is the length of the piston and $k_{p}$ is the permeability of the piston. This flux also equals the flux from the pack:

$$
q=-\left.\frac{k}{\eta} \frac{\partial P}{\partial z}\right|_{z=H}
$$

By taking the continuity of the flux, the boundary condition of the top of the pack becomes

$$
\left.\left(\frac{\partial P}{\partial z}+\frac{k_{p}}{k L_{p}} P\right)\right|_{z=H}=0
$$

By taking the dimensionless form of the variables,

$$
\Theta=\frac{P}{\sigma_{0}}, \quad Z=\frac{z}{H}, \quad T_{D}=\frac{t c_{v}}{H^{2}}, \quad C_{d}=\frac{k_{p} H}{k L_{p}},
$$

the governing equation for excess pore pressure $P=$ $P(t, z)$ becomes

$$
\frac{\partial \Theta}{\partial T_{D}}=\frac{\partial^{2} \Theta}{\partial Z^{2}},
$$

with initial condition: $\left.\Theta\right|_{T_{D}=0}=1$,

and boundary conditions: $\left.\quad \frac{\partial \Theta}{\partial Z}\right|_{Z=0}=0$,

$$
\left.\left(\frac{\partial \Theta}{\partial Z}+C_{d} \Theta\right)\right|_{z=1}=0 .
$$

We refer to the constant $C_{d}$ as the permeability ratio, which compares the permeability and diffusion distance of the two layers. Here is how the Robin boundary condition converges to other boundary conditions when $C_{d}$ approaches 0 and $\infty$ :

$$
C_{d} \begin{cases}\rightarrow 0, & \text { no flow boundary, } \\ \sim 1, & \text { comparable permeability } \\ \rightarrow \infty, & \text { constant pressure boundary }\end{cases}
$$

Equation (B8) can be solved using the separation of variables:

$$
\Theta(T, Z)=\sum_{n=1}^{n=\infty} C_{n} \cos \left(\lambda_{n} Z\right) e^{-\lambda_{n}^{2} T_{D}},
$$

$$
\begin{gathered}
C_{n}=\frac{\int_{0}^{1} \cos \left(\lambda_{n} Z\right) d z}{\int_{0}^{1} \cos ^{2}\left(\lambda_{n} Z\right) d z}=\frac{4 \sin \left(\lambda_{n}\right)}{2 \lambda_{n}+\sin \left(2 \lambda_{n}\right)}, \\
\lambda_{n}=\operatorname{Root}\left(\cot \left(\lambda_{n}\right)=\frac{1}{C_{d}} \lambda_{n}\right), \quad \lambda_{n}>0 .
\end{gathered}
$$

With the solution for the excess pore pressure, the vertical strain can be expressed as

$$
\epsilon(t, z)=\frac{\sigma_{0}-P}{m_{v}} .
$$

Taking the similar dimensionless form, the vertical strain becomes

$$
\epsilon(t, z)=\frac{\sigma_{0}}{m_{v}}(1-\Theta) .
$$

Integrating the vertical strain yields the vertical displacement $\Delta H$ :

$$
\Delta H(t)=\int_{0}^{H} \epsilon(t, z) d z=H \frac{\sigma_{0}}{m_{v}} \int_{0}^{1}(1-\Theta) d Z .
$$

The integral $\int_{0}^{1}(1-\Theta) d Z$ shows the overall dissipation of the excess pore pressure. It is also referred to as the degree of consolidation, where 0 indicates the initial state and 1 indicates the complete dissipation of pore pressure. When the degree of consolidation is 1, the final deformation $\Delta H_{T}$ is $H \sigma_{0} / m_{v}$. Equation (B13) also shows that the degree of consolidation can be measured experimentally by measuring the deformation of the pack $\Delta H$ :

$$
\int_{0}^{1}(1-\Theta) d Z=\frac{\Delta H}{H} \frac{m_{v}}{\sigma_{0}}=\frac{\Delta H}{\Delta H_{T}} .
$$

\section{APPENDIX C: EXPERIMENTAL PARAMETERS}

TABLE I. Experimental parameters.

\begin{tabular}{lcc}
\hline \hline Symbol & Value & Unit \\
\hline$d_{s}$ & 2.0 & $\mathrm{~mm}$ \\
$H$ & 20.0 & $\mathrm{~mm}$ \\
$h_{p}$ & 2.5 & $\mathrm{~mm}$ \\
$T$ & 2.1 & $\mathrm{~mm}$ \\
$L$ & 100.0 & $\mathrm{~mm}$ \\
$k$ & $3.0 \times 10^{-9}$ & $\mathrm{~m}^{2}$ \\
$k_{p}$ & $1.2 \times 10^{-8}$ & $\mathrm{~m}^{2}$ \\
$L_{p}$ & 94.0 & $\mathrm{~mm}$ \\
$M$ & 300 & $\mathrm{kPa}$ \\
$W$ & 5.0 & $\mathrm{~N}$ \\
$W_{p}$ & 0.23 & $\mathrm{~N}$ \\
$A_{p}$ & $1.8 \times 10^{-4}$ & $\mathrm{~m}$ \\
$\eta$ & $10000,5000,1000,500,50$ & $\mathrm{cSt}$ \\
\hline \hline
\end{tabular}


[1] K. Terzaghi, Erdbaumechanik auf Bodenphysikalischer Grundlage (F. Deuticke, Leipzig und Wien, 1925).

[2] K. Terzaghi, Theoretical Soil Mechanics (John Wiley \& Sons, Ltd, New York, US, 1943), Chap. 13, p. 265.

[3] A. Jain and R. Juanes, Preferential mode of gas invasion in sediments: Grain-scale mechanistic model of coupled multiphase fluid flow and sediment mechanics, J. Geophys. Res. Solid Earth 114, B08101 (2009).

[4] B. Sandnes, E. Flekkøy, H. Knudsen, K. Måløy, and H. See, Patterns and flow in frictional fluid dynamics, Nat. Commun. 2, 288 (2011).

[5] H. Huang, F. Zhang, P. Callahan, and J. Ayoub, Granular Fingering in Fluid Injection Into Dense Granular Media in a Hele-Shaw Cell, Phys. Rev. Lett. 108, 258001 (2012).

[6] R. Holtzman, M. L. Szulczewski, and R. Juanes, Capillary Fracturing in Granular Media, Phys. Rev. Lett. 108, 264504 (2012).

[7] C. W. MacMinn, E. R. Dufresne, and J. S. Wettlaufer, FluidDriven Deformation of a Soft Granular Material, Phys. Rev. X 5, 011020 (2015).

[8] M. M. Frocht, Photoelasticity (John Wiley \& Sons, New York, US, 1941).

[9] R. R. Hartley and R. P. Behringer, Logarithmic rate dependence of force networks in sheared granular materials, Nature 421, 928 (2003).

[10] T. S. Majmudar and R. P. Behringer, Contact force measurements and stress-induced anisotropy in granular materials, Nature 435, 1079 (2005).

[11] D. Bi, J. Zhang, B. Chakraborty, and R. P. Behringer, Jamming by shear, Nature 480, 355 (2011).

[12] K. E. Daniels, J. E. Kollmer, and J. G. Puckett, Photoelastic force measurements in granular materials, Rev. Sci. Instrum. 88, 051808 (2017).

[13] A. Abed-Zadeh, J. Barés, T. A. Brzinski, K. E. Daniels, J. Dijksman, N. Docquier, H. O. Everitt, J. E. Kollmer, O. Lantsoght, D. Wang, M. Workamp, Y. Zhao, and H. Zheng, Enlightening force chains: A review of photoelasticimetry in granular matter, Granul. Matter 21, 83 (2019).

[14] A. Shukla, Dynamic photoelastic studies of wave propagation in granular media, Opt. Lasers Eng. 14, 165 (1991).

[15] A. J. Rosakis, O. Samudrala, and D. Coker, Cracks faster than the shear wave speed, Science 284, 1337 (1999).

[16] L. Vanel, D. Howell, D. Clark, R. Behringer, and E. Clément, Memories in sand: Experimental tests of construction history on stress distributions under sandpiles, Phys. Rev. E 60, R5040(R) (1999).

[17] J. Geng, D. Howell, E. Longhi, R. Behringer, G. Reydellet, L. Vanel, E. Clément, and S. Luding, Footprints in Sand: The Response of a Granular Material to Local Perturbations, Phys. Rev. Lett. 87, 035506 (2001).

[18] K. Xia, A. J. Rosakis, H. Kanamori, and J. R. Rice, Geophysics: Laboratory earthquakes along inhomogenous faults: Directionality and supershear, Science 308, 681 (2005).

[19] T. S. Majmudar, M. Sperl, S. Luding, and R. P. Behringer, Jamming Transition in Granular Systems, Phys. Rev. Lett. 98, 058001 (2007).

[20] K. E. Daniels and N. W. Hayman, Force chains seismogenic faults visualized with photoelastic granular shear experiments, J. Geophys. Res. Solid Earth 113, B11411 (2008).

[21] I. Zuriguel and T. Mullin, The role of particle shape on the stress distribution in a sandpile, Proc. R. Soc. London, Ser. A 464, 2089 (2008).

[22] N. W. Hayman, L. Ducloué, K. L. Foco, and K. E. Daniels, Granular controls on periodicity of stick-slip events: Kinematics and force-chains in an experimental fault, Pure Appl. Geophys. 168, 2239 (2011).

[23] G. Huillard, X. Noblin, and J. Rajchenbach, Propagation of acoustic waves in a one-dimensional array of noncohesive cylinders, Phys. Rev. E 84, 016602 (2011).

[24] A. H. Clark, L. Kondic, and R. P. Behringer, Particle Scale Dynamics in Granular Impact, Phys. Rev. Lett. 109, 238302 (2012).

[25] E. Kolb, C. Hartmann, and P. Genet, Radial force development during root growth measured by photoelasticity, Plant Soil 360, 19 (2012).

[26] D. M. Wendell, K. Luginbuhl, J. Guerrero, and A. E. Hosoi, Experimental investigation of plant root growth through granular substrates, Exp. Mech. 52, 945 (2012).

[27] J. G. Puckett and K. E. Daniels, Equilibrating Temperaturelike Variables in Jammed Granular Subsystems, Phys. Rev. Lett. 110, 058001 (2013).

[28] J. Ren, J. A. Dijksman, and R. P. Behringer, Reynolds Pressure and Relaxation in a Sheared Granular System, Phys. Rev. Lett. 110, 018302 (2013).

[29] S. Farhadi and R. P. Behringer, Dynamics of Sheared Ellipses and Circular Disks: Effects of Particle Shape, Phys. Rev. Lett. 112, 148301 (2014).

[30] S. Lherminier, R. Planet, G. Simon, L. Vanel, and O. Ramos, Revealing the Structure of a Granular Medium through Ballistic Sound Propagation, Phys. Rev. Lett. 113, 098001 (2014).

[31] D. A. Geller, R. E. Ecke, K. A. Dahmen, and S. Backhaus, Stick-slip behavior in a continuum-granular experiment, Phys. Rev. E 92, 060201(R) (2015).

[32] N. Iikawa, M. Bandi, and H. Katsuragi, Sensitivity of Granular Force Chain Orientation to Disorder-Induced Metastable Relaxation, Phys. Rev. Lett. 116, 128001 (2016).

[33] N. Mahabadi and J. Jang, The impact of fluid flow on force chains in granular media, App. Phys. Lett. 110, 041907 (2017).

[34] S. Lherminier, R. Planet, V. L. dit Vehel, G. Simon, L. Vanel, K. J. Måløy, and O. Ramos, Continuously Sheared Granular Matter Reproduces in Detail Seismicity Laws, Phys. Rev. Lett. 122, 218501 (2019).

[35] Y. Zhao, J. Barés, H. Zheng, J. E. Socolar, and R. P. Behringer, Shear-Jammed, Fragile, and Steady States in Homogeneously Strained Granular Materials, Phys. Rev. Lett. 123, 158001 (2019).

[36] C. H. Liu, S. R. Nagel, D. A. Schecter, S. N. Coppersmith, S. Majumdar, O. Narayan, and T. A. Witten, Force fluctuations in bead packs, Science 269, 513 (1995).

[37] P. Yu, S. Frank-Richter, A. Börngen, and M. Sperl, Monitoring three-dimensional packings in microgravity, Granul. Matter 16, 165 (2014). 
[38] R. Hurley, S. Hall, J. Andrade, and J. Wright, Quantifying Interparticle Forces and Heterogeneity in 3D Granular Materials, Phys. Rev. Lett. 117, 098005 (2016).

[39] M. Workamp, S. Alaie, and J. A. Dijksman, Coaxial air flow device for the production of millimeter-sized spherical hydrogel particles, Rev. Sci. Instrum. 87, 125113 (2016).

[40] L. Wang, Y. Ju, H. Xie, G. Ma, L. Mao, and K. He, The mechanical and photoelastic properties of 3D printable stress-visualized materials, Sci. Rep. 7, 10918 (2017).

[41] A. Abed-Zadeh, J. Barés, T. Brzinski, K. Daniels, J. Dijksman, N. Docqiuer, H. Everitt, J. Kollmer, O. Lantsoght, D. Wang, M. Workamp, Y. Zhao, and H. Zheng, Photoelastic methods wiki. https://git-xen.lmgc.univ-montp2.fr/Photo Elasticity/Main/-/wikis/home (2019).

[42] D. B. Richardson, T. Z. Blanzymski, E. N. Gregory, A. R. Hutchinson, and L. M. Wyatt, in Mechanical Engineer's Reference Book, edited by E. H. Smith (ButterworthHeinemann, Oxford, UK, 1994), 12th ed., p. 16/105.

[43] ASM International, Practical Guide to Image Analysis (ASM International, Materials Park, Ohio, US, 2000).

[44] M. Workamp, S. Alaie, and J. A. Dijksman, What is fluidity? Designing an experimental system to probe stress and velocity fluctuations in flowing suspensions, EPJ Web Conf. 140, 03020 (2017).

[45] B. E. Sørensen, A revised Michel-Lévy interference colour chart based on first-principles calculations, Eur. J. Mineral 25, 5 (2013).

[46] See Supplemental Material at http://link.aps.org/supple mental/10.1103/PhysRevApplied.16.024043 for videos of calibration tests and a consolidation test.

[47] A. Abed-Zadeh, J. Barés, and R. P. Behringer, Crackling to periodic dynamics in granular media, Phys. Rev. E 99, 040901(R) (2019).

[48] S. Westland, C. Ripamonti, and V. Cheung, in Computational Colour Science using MATLAB ${ }^{\circledR}$ (John Wiley \& Sons, Chichester, UK, 2012), Chap. 5, p. 49.

[49] T. W. Lambe and R. V. Whitman, Soil Mechanics (John Wiley \& Sons, New York, US, 1969).

[50] P. C. Carman, Fluid flow through granular beds, Trans. IChemE 15, 150 (1937).

[51] N. Lu and W. J. Likos, Unsaturated Soil Mechanics (Wiley, Hoboken, N.J., US, 2004).

[52] R. Juanes, Y. Meng, and B. K. Primkulov, Multiphase flow and granular mechanics, Phys. Rev. Fluids 5, 110516 (2020).
[53] M. Scheel, R. Seemann, M. Brinkmann, M. Di Michiel, A. Sheppard, B. Breidenbach, and S. Herminghaus, Morphological clues to wet granular pile stability, Nat. Mater. 7, 189 (2008).

[54] H. Shin and J. C. Santamarina, Fluid-driven fractures in uncemented sediments: Underlying particlelevel processes, Earth Planet Sci. Lett. 299, 180 (2010).

[55] H. J. Cho, N. B. Lu, M. P. Howard, R. A. Adams, and S. S. Datta, Crack formation and self-closing in shrinkable, granular packings, Soft Matter 15, 4689 (2019).

[56] Y. Meng, B. K. Primkulov, Z. Yang, C. Y. Kwok, and R. Juanes, Jamming transition and emergence of fracturing in wet granular media, Phys. Rev. Res. 2, 022012(R) (2020).

[57] A. Skarke, C. Ruppel, M. Kodis, D. Brothers, and E. Lobecker, Widespread methane leakage from the sea floor on the northern us atlantic margin, Nat. Geosci. 7, 657 (2014).

[58] B. P. Scandella, K. Delwiche, H. F. Hemond, and R. Juanes, Persistence of bubble outlets in soft, methane-generating sediments, J. Geophys. Res. Biogeosci. 122, 1298 (2017).

[59] S. Lee, J. Lee, R. Le Mestre, F. Xu, and C. W. MacMinn, Migration, trapping, and venting of gas in a soft granular material, Phys. Rev. Fluids 5, 084307 (2020).

[60] P. Adler and P. Mills, Motion and rupture of a porous sphere in a linear flow field, J. Rheol. 23, 25 (1979).

[61] J. Guery, E. Bertrand, C. Rouzeau, P. Levitz, D. Weitz, and J. Bibette, Irreversible Shear-Activated Aggregation in Non-Brownian Suspensions, Phys. Rev. Lett. 96, 198301 (2006).

[62] B. O. Conchúir and A. Zaccone, Mechanism of flowinduced biomolecular and colloidal aggregate breakup, Phys. Rev. E 87, 032310 (2013).

[63] Y. Guglielmi, F. Cappa, J.-P. Avouac, P. Henry, and D. Elsworth, Seismicity triggered by fluid injection-induced aseismic slip, Science 348, 1224 (2015).

[64] M. Scuderi, C. Collettini, and C. Marone, Frictional stability and earthquake triggering during fluid pressure stimulation of an experimental fault, Earth Planet Sci. Lett. 477, 84 (2017).

[65] M. Alghannam and R. Juanes, Understanding rate effects in injection-induced earthquakes, Nat. Commun. 11, 3053 (2020). 